\title{
The Effect of Surfactant Choice and Concentration on the Dimensions and Yield of Liquid Phase Exfoliated Nanosheets
}

Aideen Griffin, ${ }^{1}$ Katharina Nisi, ${ }^{2}$ Joshua Pepper, ${ }^{1}$ Andrew Harvey, ${ }^{1}$ Beata M. Szydłowska, ${ }^{2}$ Jonathan N. Coleman ${ }^{1}$, Claudia Backes ${ }^{2}$ *

${ }^{1}$ School of Physics and CRANN \& AMBER Research Centres, Trinity College Dublin, Dublin 2, Ireland

${ }^{2}$ Physical Chemistry Institute, Ruprecht-Karls University Heidelberg, Im Neuenheimer Feld 253, 69120 Heidelberg, Germany

*backes@uni-heidelberg.de

\begin{abstract}
:
Liquid phase exfoliation has progressed in recent years to become a common method of production for $2 \mathrm{D}$ materials. During exfoliation, surfactants can be used to stabilize the nanosheets against reaggregation. Here, using $\mathrm{WS}_{2}$ as a model system, we explore the effect of varying surfactant type and concentration on the yield and dimensions of the exfoliated nanosheets. For ionic surfactants, the mass of nanosheets produced was constant for low surfactant concentrations but decreased sharply for surfactant concentrations above $\sim 10 \mathrm{mM}$, regardless of surfactant. Very similar surfactant-concentration dependence was observed for both nanosheet length and thickness. Contrary to previous reports, this data implies that the optimum surfactant concentration is not linked to the critical micelle concentration. In addition, we found that surfactant concentrations as low as $0.07 \mathrm{mM}$ yielded stable nanosheet dispersions with zeta potential above $40 \mathrm{mV}$. By decoupling the exfoliation and stabilization effects of the surfactant, we have shown that it is the (de)stabilization process, rather than the exfoliation process, which links nanosheet concentration, size and thickness to surfactant concentration.
\end{abstract}




\section{Introduction}

One of the most common and probably the most versatile methods for production of $2 \mathrm{D}$ materials is liquid phase exfoliation (LPE). ${ }^{1}$ This method involves the sonication or shearing of layered crystal to generate 2D nanosheets which can be stabilized in a range of liquid environments. ${ }^{2-7}$ In this way, LPE has been used to exfoliate a wide range of $2 \mathrm{D}$ materials including graphene, ${ }^{8-10} \mathrm{BN},{ }^{11} \mathrm{GaS},{ }^{12}$ phosphorene ${ }^{13}$ and MXenes ${ }^{14}$ and of course a number of transition metal dichalcogenides (TMDs) including $\mathrm{MoS}_{2}{ }^{15}, 16$ This scalable, low cost technique allows for stabilization either thermodynamically using appropriate solvents or electrostatically with surfactants. ${ }^{2,17}$ In particular, surfactant exfoliation is attractive as all processing is done in aqueous environments. While various surfactants have been used as stabilizers during liquid phase exfoliation, the effect of surfactant choice and concentration on nanosheet yield and dimensions has yet to be explored comprehensively.

Although binding of particular surfactants such as sodium dodecyl sulfate to graphite has been examined in detail, ${ }^{18}$ studies of surfactant-stabilized nanosheets are limited and have been performed mainly for graphene. ${ }^{19-22}$ Ionic surfactants are the most commonly studied with sodium cholate (SC), sodium dodecyl sulfate (SDS) and other long alkyl chain surfactants at the forefront. ${ }^{17,22-25}$ In broad terms, stabilization of nanosheets (and colloids in general) using ionic surfactants is relatively simple, though the surfactant conformation on the nanosheet surface in solution requires further exploration, with various binding orientations and mechanisms suggested. ${ }^{20,23-27}$ The surfactant tail groups (or nonpolar part of the molecule in general) tend to adsorb on the nanosheet surface yielding a layer of bound charge which is compensated by a diffuse, semi-mobile cloud of counterions associated with the head groups. The resultant double layer acts as an electrical multipole and, from a distance, appears to carry a net charge. Thus, adjacent surfactant-coated nanosheets experience an electrostatic repulsion which compensates their innate van der Waals attraction and prevents aggregation. ${ }^{28}$ However, the fine details of this mechanism would be expected to depend on both nanosheet and surfactant type and are not well-studied.

Surfactants can be classified based on their ionicity into three different types; cationic, anionic and non-ionic. Most ionic surfactants are composed of a long hydrophobic tail (typical alkyl chains) and a charged headgroup. However, other structural motifs can lead to amphiphilicity. For example, facial amphiphiles are composed of a rigid molecular framework with a polar and nonpolar side and thus exhibit planar polarity. These tend to form smaller micelles at a higher critical micelle concentration than conventional surfactants. ${ }^{29-31}$ 
Sodium cholate, one of the most commonly used surfactants for LPE is such a facial amphiphile. The molecule has been reported to lie flat on the surface of graphene with its hydroxyl and carboxyl groups orientated towards the aqueous solution ${ }^{20,26}$ yielding stable graphene dispersions due to the molecule's geometric similarity with graphene's hydrophobic surface. ${ }^{19}$ Steric repulsion contributes significantly to the stabilization mechanism in this case. $^{24}$

In contrast, conventional amphiphiles such as sodium dodecyl sulfate on graphitic surfaces, can adsorb in a head-to-tail or tails-on configuration, respectively, depending on the surfactant concentration. ${ }^{27}$ Similarly, for the ionic sodium dodecyl sulfate and other surfactants with varying alkyl chain length, the stability of graphene dispersions below a chain length of 16 (i.e. those studied in this work) is noted to be largely due to mean field electrostatics from the ionic nature of the surfactants and their effective surface charge densities with steric factors being of minor impact. ${ }^{20}$

We would therefore expect that the efficiency of the stabilization of LPE nanosheets depends crucially on surfactant concentration and chemical structure. However, no systematic work was performed, where not only nanosheet concentration, but also lateral size and thickness was investigated as a function of surfactant concentration. For such a study, TMDs are ideal model systems due to recently established spectroscopic metrics to reliably assess concentration and nanosheet dimensions. ${ }^{5,32}$

Studies on surfactant-stabilized TMDs have largely been limited to $\mathrm{MoS}_{2}$ with a small number of surfactants, namely SDS and cationic $\mathrm{CTAB},{ }^{23,25}$ and focused on the relationship between zeta potentials and surfactant double layer coating the nanosheet. ${ }^{23}$ In $\mathrm{MoS}_{2}-\mathrm{CTAB}$ and $\mathrm{MoS}_{2}-\mathrm{SDS}$ dispersions, the bound and free surfactant chains are reported to undergo rapid exchange ${ }^{23}$ arranging themselves flat on the surface of the nanosheet in a random fashion. More recent studies from the same authors ${ }^{25}$ investigating $\mathrm{MoS}_{2}$ in $\mathrm{CTAB}$, note that ions in solution cannot access the $\mathrm{MoS}_{2}$ surface because of hydrophobic CTAB cation chains and that zeta potential depends on both surface charge and on the ionic strength of the media. CTAB produced a similar magnitude and effective surface charge distribution with only the sign of the zeta potential differing from $\mathrm{MoS}_{2}$ - SDS dispersions. ${ }^{23}$ Consequently, we would predict the absolute value of the zeta potential for TMDs would not be significantly different for cationic and anionic surfactants. 
More extensive studies have been completed on carbon nanotube (CNT)-surfactant systems. ${ }^{33-42}$ While it is possible to disperse in concentrations below the critical micelle concentration (CMC), ${ }^{38}$ surfactants generally disperse CNTs better at higher concentrations than those typically used in 2D LPE dispersions. ${ }^{33-35,37}$ At very high surfactant concentration $(>10 \times$ $\mathrm{cmc}^{42}$ ), it has been reported that there is a reduction in CNT concentration due to attractive depletion interactions. ${ }^{35,42-45}$ These concentrations are dependent on nature of the surfactant molecule. Hydrophobic tails in ionic surfactants are thought to adsorb to the nanotube surface with stabilization facilitated by electrostatic interactions. ${ }^{46}$ Aromatic molecules, for example sodium dodecylbenzene sulphonate, can increase the adsorption rate to the nanotube surface through $\pi$-stacking interactions of the benzene ring. ${ }^{37,42}$ Moreover, an increase in surfactant chain length leads to an increase in nanosheet dispersibility, as a result of enhanced hydrophobicity from longer-chained surfactants. ${ }^{42}$ Anionic surfactants in general, have proved more effective than cationics in dispersing CNTs as found experimentally by Blanch et al. ${ }^{35}$ and Fernandes et al. ${ }^{42}$ and investigated theoretically by Xu et al. ${ }^{36}$. Indeed White et al. ${ }^{33}$ demonstrated that there is a preferential binding of SDS over CTAB in nanotube dispersions. It has been suggested that the $\mathrm{Na}^{+}$counterion in the anionic surfactant balances the electrostatic forces. ${ }^{36}$ Overall, this strongly suggests that dispersed concentration and dispersion quality (i.e. degree of debundling or exfoliation, respectively) is dependent on surfactant concentration and type.

Given the limited exploration of surfactant effects with TMDs, we aim to investigate if there is indeed an effect on the properties of nanosheets produced by liquid exfoliation due to the type and concentration of surfactant. We focused on simple alkyl sulfates and bile salts in this work, where we do not expect any specific chemical interactions to occur. Other types of stabilizers have been used for LPE, including pyrene-based derivatives ${ }^{47-49}$, in which strong adsorption occurs through $\pi-\pi$ interactions of the planar pyrene based surfaces with for example graphene. Here, we have used a broad range of commercially available standard surfactants to examine the role of surfactant type and its respective concentration on the resulting nanosheet dispersion, specifically nanosheet dimensions and concentration. We use $\mathrm{WS}_{2}$ as a model system stabilized with twelve different surfactants of varying ionicity and molecular weight (i.e. chain length) (SI.1). Through spectroscopy and zeta potential measurements, we investigate the effect of each surfactant on the concentration as well as size and thickness of the exfoliated nanosheets. 


\section{Results and Discussion}

The main aim of this work is to identify the effect of surfactant choice and concentration on the yield, length and thickness of nanosheets produced by LPE. To achieve this, we exfoliated $\mathrm{WS}_{2}$ in a range of surfactants, each at a number of different surfactant concentrations, while using the same exfoliation and centrifugation conditions for each sample (for details see Methods and SI). The surfactants used were sodium cholate (SC), sodium deoxycholate (SDC), sodium dodecyl sulfate (SDS), sodium tetradecyl sulfate (STS), sodium octyl sulfate (SOS), lithium dodecyl sulfate (LDS), sodium dodecylbenzene sulphonate (SDBS), cetyltrimethylammonium bromide (CTAB), tetradecyltrimethylammonium bromide (TTAB), (for details on each surfactant see SI.9). Each sample was sonicated for $2 \mathrm{~h}$ with a tapered tip and then centrifuged in two steps to remove the largest and smallest material (i.e. centrifuged at $106 \mathrm{~g}$ to remove large material and $21,130 \mathrm{~g}$ to remove small material, each for $2 \mathrm{~h}$ ). For each surfactant, nanosheets were exfoliated in surfactant solutions using 4-16 different surfactant concentrations within the global range of $0.03-30 \mathrm{gL}^{-1}$. In addition, graphene and $\mathrm{MoS}_{2}$ were exfoliated in SC for comparison. Examples of dispersions of $\mathrm{WS}_{2}$ nanosheets exfoliated in solutions of SC at varying concentrations are displayed in Fig1A, with each showing the typical dark green colour expected for $\mathrm{WS}_{2}$ nanosheets.

While liquid phase exfoliation is a versatile method of exfoliation, it yields polydisperse samples, especially when the dispersions are not size-selected, as was the case here. A selection of samples were characterized microscopically using both TEM and AFM to confirm production of nanosheets of similar lateral size and thickness as reported previously. ${ }^{5,32} \mathrm{~A}$ sample TEM image of $\mathrm{WS}_{2}$ nanosheets produced in sodium cholate surfactant $\left(2 \mathrm{~g} \mathrm{~L}^{-1}\right)$ is represented in Fig 1B showing nanosheets with lateral sizes in the range 50 to $400 \mathrm{~nm}$ and clearly illustrating the polydispersity of the nanosheets produced by LPE. AFM images such as that displayed in Fig 1C show a broad nanosheet thickness variation with nanosheets varying from monolayers to 30-40 layers, consistent with previous reports for $\mathrm{WS}_{2}$ in sodium cholate surfactant $\left(2 \mathrm{~g} \mathrm{~L}^{-1}\right)^{32}$.

While nanosheet size and thickness can of course be measured accurately by AFM (at least when using appropriate corrections for length and layer number ${ }^{50}$ ), such experiments are very time consuming. One reason for choosing $\mathrm{WS}_{2}$ as a model system is the fact that, for this material, nanosheet size and thickness, as well as concentration, can be extracted quickly and easily from optical spectra. ${ }^{7,} 32$ To achieve this, optical extinction spectra were measured for 
each dispersion (extinction, Ext, is related to the transmission, $\mathrm{T}$, via $\mathrm{T}=10^{-\mathrm{Ext}}$ with $\mathrm{Ext}=\varepsilon \mathrm{C} \ell$ where $\varepsilon$ is the extinction coefficient, $\mathrm{C}$ the concentration and $\ell$ the path length). All extinction spectra were measured under the same conditions using their respective surfactant solutions as a baseline. Using previously established metrics, ${ }^{51}$ we can estimate the nanosheet concentration as well as mean nanosheet length and thickness from these extinction spectra (see SI section 2).

To illustrate the dependence of extinction spectra on such parameters, we plot a set of extinction spectra for $\mathrm{WS}_{2}$ exfoliated in sodium cholate at a range of SC concentrations in figure $2 \mathrm{~A}$. For clarity, we show the same spectra normalized to the extinction at $294 \mathrm{~nm}$ in figure 2B. It is clear from this figure that the spectra change in a number of ways as the SC concentration is varied. For example, the absolute magnitude of extinction varies with SC concentration (figure $2 \mathrm{~A})$. This indicates that the concentration of $\mathrm{WS}_{2}$ nanosheets changes as the surfactant concentration is varied. In addition, figure $2 \mathrm{~B}$ clearly indicates that the overall spectral shape differs significantly with surfactant concentration. This is due to variations in the contribution of nanosheet edges versus basal planes to the extinction coefficient on the one hand and varying contributions from light scattering on the other. This demonstrates that the mean nanosheet lateral size is dependent on the surfactant concentration. ${ }^{5,51}$ Finally, as seen in figure 2B inset, the position of the A-exciton around $650 \mathrm{~nm}$ shifted subtly with surfactant concentration. This is due to confinement and dielectric screening effects of the exciton and indicates that the mean nanosheet thickness varies with SC concentration. ${ }^{5,51}$ In fact, the effects outlined above mean that the extinction spectra can be used to quantitatively estimate the nanosheet concentration, length and thickness. A detailed explanation of this procedure is given in the SI.

\section{Dependence of nanosheet concentration and dimensions on the SC concentration}

Prior to comparing the data obtained from exfoliation and stabilization in different surfactants, it is important to verify the suitability of sample preparation and data analysis. To this end, we first focus on the data of $\mathrm{WS}_{2}$ exfoliated in sodium cholate. In our sample preparation procedure, we have implemented two steps that we find crucial. Firstly, we performed a precleaning step by sonicating the starting material in water to remove impurities. As shown in the SI (section 3), these impurities can have an impact on the accuracy of the UV-Vis metrics on the one hand. On the other hand, we found that they are ionic in nature which can have an impact on the colloidal stability using ionic surfactants. A plot of ionic conductivity as function of SC concentration in figure 3A shows a linear scaling (dashed line is a fit through the origin) 
above an SC concentration of $0.5 \mathrm{gL}^{-1}$ which is a strong indication that the electrostatic interactions in the dispersion are governed by the surfactant concentration with remaining traces of ionic impurities influencing the data at SC concentrations below $0.5 \mathrm{gL}^{-1}$.

Secondly, in addition to a data analysis based on UV/Vis, we also targeted to analyze photoluminescence and Raman spectra of the samples to gain insights in monolayer population. ${ }^{3}$ Such measurements are greatly facilitated in high concentration dispersions, so that we implemented a centrifugation step at relatively high centrifugal acceleration $(21 \mathrm{k} g)$ to pellet out the nanosheets which enabled redispersing them at reduced volume, i.e. higher concentration. On redispersion of this sediment, the same surfactant concentration was used as in the exfoliation. However, this sedimentation might have a higher local concentration of surfactant trapped between the nanosheets in the precipitate than the nominal surfactant concentration used on redispersion. To test whether there is a major impact on the overall data discussed below, a couple of reference experiments were performed where this high speed centrifugation step was omitted. The data is included in figure 3 as red stars and confirms that potentially trapped surfactant has a negligible impact. That this data collapses on the same curve as the other data points in the plot of ionic conductivity as function of surfactant concentration confirms that i) the amount of potentially trapped surfactant is low, ii) the presonication treatment widely removes ionic impurities, as these would still be present in the analyzed supernatant samples, but not the redispersed sediments.

As mentioned above, we can extract information on dispersed concentration as well as average lateral size $(<\mathrm{L}\rangle)$ and layer number $(<\mathrm{N}\rangle)$ from the extinction spectra. This data is summarized using SC as surfactant in figure 3B-D (for an explanation of error bars see SI section 4). The analysis of the dispersed nanosheet concentration in figure $3 \mathrm{~B}$ first shows an increase in nanosheet concentration with increasing surfactant concentration below $\mathrm{CSC}_{\mathrm{SC}} \mathrm{gL}^{-1}$. Intuitively, this behavior can be inferred by assuming that the nanosheet concentration is maximized when approaching the maximum surfactant coverage. However, above this surfactant concentration, we observe a striking decrease in the concentration of dispersed sheets. At the same time, both $\langle\mathrm{N}\rangle$ (Figure 3C) and $\langle\mathrm{L}\rangle$ (Figure 3D) decrease at the same threshold concentration even though these parameters were widely invariant below $C_{S C} 2 \mathrm{gL}^{-1}$. The remainder of the discussion and data analysis of this manuscript will focus on this aspect which we will ultimately identify as destabilization of nanosheets due to electrostatic screening at high surfactant concentration. The changes in both $\langle\mathrm{N}\rangle$ and $\langle\mathrm{L}\rangle$ with increasing surfactant concentration observed in Figures 3C-D certainly point towards an in situ control of nanosheet 
dimensions during exfoliation. It is thus important to track down in which way this is dependent on the surfactant type and how this surfactant concentration threshold can be rationalized.

\section{Dependence of nanosheet concentration on surfactant concentration for various stabilizers}

As outlined above, through analysis of the extinction spectra, we can extract the nanosheet concentration which is plotted versus surfactant concentration, $\mathrm{C}_{\text {surf, }}$ expressed both as $\mathrm{g} \mathrm{L}^{-1}$ and $\mathrm{mM}$ in figures $4 \mathrm{~A}$ and $\mathrm{B}$ across the range of surfactant used. In contrast to the data in figure 3 , we now plot the result of the analysis on a log-log plot to observe the changes occurring at high $\mathrm{c}_{\text {surf }}$ more clearly. We note that graphs Fig. 4A and 4B look similar because of the limited ranged of surfactant molecular weights under study. These graphs include data for $\mathrm{WS}_{2}$ exfoliated in 9 ionic surfactants as well as $\mathrm{MoS}_{2}$ and graphene exfoliated in SC, all prepared by exfoliating layered powder with starting concertation of $20 \mathrm{~g} \mathrm{~L}^{-1}$. For comparison, we include data for $\mathrm{WS}_{2}$ exfoliated in SDS prepared with two different starting concentration of $\mathrm{WS}_{2}\left(10\right.$ and $\left.40 \mathrm{~g} \mathrm{~L}^{-1}\right)$. For these latter two $\mathrm{WS}_{2}$ samples, the data in Fig $4 \mathrm{~A}$ and $\mathrm{B}$ were corrected for the different starting concentration (i.e. multiplied by 2 and 0.5 respectively) to allow better comparison with the rest of the dataset (for actual values see SI.5). For data on non-ionic surfactants see SI.6. Interestingly, all data points appear to fall roughly on master curves, irrespective of the surfactant. At low surfactant concentration, the nanosheet concentration is roughly constant at $1-2 \mathrm{~g} \mathrm{~L}^{-1}$. This is consistent with previous work showing the concentration of surfactant-stabilized graphene to vary only weakly with surfactant choice. ${ }^{52}$ However, we observe a sharp decrease in nanosheet concentration for surfactant concentrations above a critical value of $\sim 5 \mathrm{~g} \mathrm{~L}^{-1}(10 \mathrm{mM})$, falling as low as $0.05 \mathrm{~g} \mathrm{~L}^{-1}$ for surfactant concentrations of $30 \mathrm{~g} \mathrm{~L}^{-1}(\sim 70 \mathrm{mM})$. We hypothesize that, above this critical concentration $(10 \mathrm{mM})$, depletion interactions ${ }^{53,54}$ may lead to the preferential flocculation of larger nanosheets leading to a decrease in nanosheet concentration.

It was originally believed that the optimal surfactant concentration, i.e. that yielding the highest concentration of nanosheets, was related to the critical micelle concentration (CMC). ${ }^{17}$ However, more recent reports have suggested that the CMC may not determine the optimum surfactant concentration for nanosheet exfoliation. ${ }^{22}$ As shown in Fig $4 \mathrm{C}$, the critical micelle concentration (CMC) for various cationic, anionic and non-ionic surfactants used here varies over a broad range, from $\sim 1$ to $130 \mathrm{mM}$. In general, the alkyl chain type surfactants (both cationic and anionic) decrease in CMC with increasing chain length. ${ }^{55,56}$ The data in figure 4 A-B appears completely independent of the CMC of the surfactant. In addition, the fact that the nanosheet concentration falls with $\mathrm{C}_{\text {surf }}$ regardless of its $\mathrm{CMC}$ is consistent with data 
reported by Wang et al. ${ }^{22}$ This suggests that, unlike nanoparticles, 2D nanosheets are not encapsulated in micelles, but that individual surfactant molecules adsorb on the surface as individual molecules irrespective of the surfactant type and concentration. We note that the sharp fall-off in nanosheet concentration above a threshold concentration of $10 \mathrm{mM}$ is not above the CMC in all cases (see for example SOS). This in contrast to depletion theory at first glance. ${ }^{53,54}$ However, we note that the local concentration around the nanosheets cannot be estimated and can very well be higher than the concentration of surfactant free in solution.

\section{Dependence of nanosheet dimensions on surfactant concentration for various stabilizers}

As indicated above, a big advantage of performing a study like this using $\mathrm{WS}_{2}$ as a model system is the ease with which nanosheet dimensions can be measured via extinction spectroscopy. For each dispersion, the mean nanosheet thickness, $\langle\mathrm{N}\rangle$, for both $\mathrm{WS}_{2}$ and $\mathrm{MoS}_{2}$ nanosheets was calculated from the A-exciton position using previously reported metrics. ${ }^{5,51}$ For graphene nanosheets mean nanosheet thickness was calculated from the ratio of extinction at two wavelengths (Ext $550 /$ Ext $\left._{325}\right)$ as reported previously. ${ }^{3}$ This thickness, expressed as number of stacked monolayers per nanosheet, is plotted versus $\mathrm{C}_{\text {surf }}$ in fig $4 \mathrm{D}$. This graph shows $\langle\mathrm{N}\rangle$ to be roughly constant at $\sim 10-15$ layers for low surfactant concentration until a surfactant concentration of about $10 \mathrm{mM}$ at which point $\langle\mathrm{N}\rangle$ decreases with increasing surfactant concentration reaching $\langle\mathrm{N}\rangle \sim 2$ layers at $\mathrm{C}_{\text {surf }} \sim 70 \mathrm{mM}$.

Similarly, the mean nanosheet length, $\left\langle\mathrm{L}>\right.$, was calculated for $\mathrm{WS}_{2}$ from the ratio of extinction at two wavelengths $(465 \mathrm{~nm}$ and $345 \mathrm{~nm}), \operatorname{Ext}_{465} / \mathrm{Ext}_{365}\left(\mathrm{Ext}_{440} / \mathrm{Ext}_{400}\right.$ for $\left.\mathrm{MoS}_{2}\right)$, again using published metrics. ${ }^{51}$ 5,32,57 We note that the length was calculated using extinction values in the visible region to avoid any effects of oxidation in the UV region (see SI.2 for details). The spectroscopic metrics used in this work were validated by statistical microscopy results shown in SI 2.4. Similar to nanosheet thickness, the mean nanosheet length (Fig 3E) remains constant at around $200 \mathrm{~nm}$ for low surfactant concentration until a surfactant concentration of about 10 $\mathrm{mM}$ at which point the length decreases, falling as low as $80 \mathrm{~nm}$. The $\mathrm{MoS}_{2}$ nanosheets appear to be even smaller $(50 \mathrm{~nm})$ for the same surfactant concentration. However this may be due to the relative error in different metrics used for each material, as the breakdown of metrics for very small or large nanosheets, respectively is material dependent (see below). For a comparison between cationic, anionic and non-ionic surfactants, see SI.7.

At this point, we must note that care must be taken when using spectroscopic metrics to measure nanosheet dimensions. The problem is that these metrics only work well over certain 
ranges in $\langle\mathrm{N}\rangle$ and $\langle\mathrm{L}\rangle$. In particular, the metric which yields $\langle\mathrm{N}\rangle$ is not expected to work well above $\langle\mathrm{N}>\sim 10$ as the nanosheets become bulk-like. This means that we would expect the A-exciton not to shift further for $\langle\mathrm{N}\rangle\rangle 10$. However, the thicker nanosheets are also laterally larger resulting in a greater contribution from light scattering to the extinction spectra ${ }^{5,32}$ Since scattering spectra are red-shifted to the absorbance spectra in the resonant regime, ${ }^{5,32}$ this can lead to artificial peak shifts of the A-exciton in extinction spectra which are no longer due to confinement and dielectric screening effects, but light scattering. To test the reliability of the $\langle\mathrm{L}\rangle$ and $\langle\mathrm{N}\rangle$ assessment, we plot $\langle\mathrm{L}\rangle^{2}$ vs. $\langle\mathrm{N}\rangle$ in figure $4 \mathrm{~F}$. It is known from a recent combined experimental and theoretical study that the mean nanosheet area should scale roughly as $\langle\mathrm{N}\rangle^{2}$ for a wide range of nanosheets. ${ }^{50}$ While this power-law is clearly present in figure $4 \mathrm{~F}$ for $\mathrm{N}<10$ (dashed line), clear deviations from this trend can be observed for larger $\langle\mathrm{N}\rangle$. This indicates that those values for $\langle\mathrm{N}\rangle$ which are larger than 10 in figure $4 \mathrm{D}$ are slightly overestimated and are in fact nearer to 10 . However, this should not change the general trend in figure $4 \mathrm{~F}$ too much as correction for this effect would just compress the scatter at low $\mathrm{C}_{\text {surf. }}$

The general relationship between $\langle\mathrm{L}\rangle$ and $\langle\mathrm{N}\rangle$ mentioned above may explain why the $\langle\mathrm{N}\rangle \mathrm{v}$ $\mathrm{C}_{\text {surf }}$ and $\langle\mathrm{L}\rangle \vee \mathrm{C}_{\text {surf }}$ curves are of similar shape. However, it is unclear why nanosheet dimensions and concentration fall off above $\mathrm{C}_{\text {surf }} \sim 10 \mathrm{mM}$. Interestingly, the trends in concentration, $\langle\mathrm{N}\rangle$ and $\langle\mathrm{L}\rangle$ when plotted versus surfactant concentration, do not appear to be material dependent with $\mathrm{MoS}_{2}$ and graphene in SC falling on the same mastercurve (SI.8).

The total number of nanosheets per volume in the initial dispersion $(\sim 20 \mathrm{mls})$ when plotted versus surfactant concentration shows a sublinear dependence, with the number of nanosheets in solution varying weakly with surfactant concentration (Fig 4G). This suggests that there may only be a certain number of nanosheets available for stabilization in a given volume. A test on the effect of stabilization versus exfoliation is described in more detail later.

A number of specific points are worth noting. Previous work has shown that the hydrophobicity of the tail group can be further increased with the removal of one (of three) oxygen atoms in the molecule resulting in the surfactant sodium deoxycholate (SDC). Authors have noted that there is an increase in the hydrophobic interactions between surfaces using SDC for graphene dispersions leading to a higher overall nanosheet yield. ${ }^{21,26}$ On the contrary, we did not see an appreciable difference in concentration or nanosheet dimension when comparing $\mathrm{WS}_{2}$ dispersions stabilised with sodium cholate and sodium deoxycholate (SI.9 for detailed comparison). 
Another more detailed comparison shows that cationic surfactants do not produce $\mathrm{WS}_{2}$ nanosheets which are quite as small or as thin as those achieved with anionic surfactant as can be seen clearly in SI.7. It is possible that the positively charged sodium ion in the anionic surfactant plays an important role in dispersion of $\mathrm{WS}_{2}$ nanosheets as suggested theoretically by $\mathrm{Xu}$ et. al for carbon nanotubes dispersed in $\operatorname{SDS}^{36}$ and by Poorsargol et al for graphene. ${ }^{58}$ The higher affinity of adsorption of smaller sodium ions to the adsorbed surfactants, compared to bulkier bromine ions from cationic surfactants, may alter the inter-nanosheet attraction, leading to smaller, thinner nanosheets (SI.7). Ultimately, this remains hard to judge due to the relatively high Krafft temperature of CTAB which is close to room temperature. ${ }^{59}$ Since it is important for such a study that all surfactant molecules are present either as individual molecules or micelles, but not crystallites, the CTAB data might generally be obscured slightly.

\section{Dependence of monolayer population on surfactant concentration for various stabilizers}

The fact that nanosheet thickness falls with increasing surfactant concentration, implies that the fraction of nanosheets which are monolayers rises with increasing $\mathrm{C}_{\text {surf. Raman }}$ spectroscopy is a relatively simple way to gain information about the monolayer population. This is because, for $\mathrm{WS}_{2}$ at the appropriate excitation wavelength $(532 \mathrm{~nm})$, the Raman spectrum contains both Raman modes at $<400 \mathrm{~cm}^{-1}$ and direct bandgap photoluminescence from monolayers $\left(\sim 2500 \mathrm{~cm}^{-1}\right)$. Because all nanosheets contribute to Raman but only monolayers contribute to PL, the ratio of PL to Raman intensities $\left(\mathrm{I}_{\mathrm{PL}} / \mathrm{I}_{\mathrm{R}}\right)$ is proportional to the monolayer volume fraction. ${ }^{51}$ Raman measurements were performed on liquid droplets of each dispersion for all surfactants and concentration. The laser was focused approximately 3-5 $\mu \mathrm{m}$ above the surface to minimize reabsorption and inner-filter effects. Normalized Raman spectra for a subset of surfactant concentrations of sodium cholate are shown in Fig 5A. The characteristic $\mathrm{WS}_{2} 2 \mathrm{LA}(\mathrm{M})$ and $\mathrm{E}_{1}{ }^{2 \mathrm{G}}(\Gamma)$ modes are present at approximately $356 \mathrm{~cm}^{-1}{ }^{60}$ Typically, in addition to these modes, a peak attributed to photoluminescence (PL) is observed at approximately $2460 \mathrm{~cm}^{-1}$. A further peak at $3400-3500 \mathrm{~cm}^{-1}$ can be attributed to water since measurements were carried out in dispersion. Shown in Fig 5B is a plot of $I_{P L} / I_{R}$ vs. surfactant concentration. The peak intensity is plotted as opposed to peak area but in line with previous work of LPE $\mathrm{WS}_{2},{ }^{61}$ we found these parameters to scale linearly with each other (SI.10), confirming the trend is still valid. This graph clearly indicates that the monolayer volume fraction increases with increasing surfactant concentration. As previously studied, ${ }^{50}$ the monolayer volume fraction scales with layer number $\langle\mathrm{N}\rangle$. As such, the scaling of $\mathrm{I}_{\mathrm{PL}} / \mathrm{I}_{\mathrm{R}}$ with 
surfactant concentration confirms the observation from the UV-Vis analysis. Due to the fact that $\langle\mathrm{N}\rangle$ varies weakly below $10 \mathrm{mM}$ (Fig $4 \mathrm{D}$ ), the $\mathrm{I}_{\mathrm{PL}} / \mathrm{I}_{\mathrm{R}}$ also levels off below $10 \mathrm{mM}$, but less abruptly which we attribute to a limited accuracy of the PL/Raman ratio to infer the monolayer content for dispersions containing predominantly few-layered sheets. Interestingly, as with the graphs in figure 4, all photoluminescence intensity data falls roughly on a master curve (albeit with more scatter) indicating that monolayer population is invariant with surfactant type. This data also implies that the scaling of the relative PL intensity with monolayer volume fraction is surprisingly robust and can be applied to a broad range of stabilisers. This is an interesting observation, as one might expect the surfactant surrounding to have an impact on the A-exciton photoluminescence response. We note that variations in PL position and widths are observed for the different surfactants. However, apparently the monolayer PL quantum yield is similar in different surfactants so that the $I_{P L} / I_{R}$ ratio is governed by the monolayer content.

\section{Dependence of Zeta potential on surfactant concentration}

Surfactant-coated nanosheets are primarily stabilized by electrostatic repulsion due to the electrical double layer associated with the bound surfactant molecules. A common measure of the strength of this repulsion is the Zeta potential. A simple rule of thumb is that, for stability against aggregation in a colloidal system, zeta potentials need to be greater than $\pm 30 \mathrm{mV}$ with higher zeta potentials leading to greater stability. We measured the zeta potential for dispersions of $\mathrm{WS}_{2}$ in all surfactants at all surfactant concentrations. While cationic and ionic surfactants gave positive and negative zeta potential respectively, for ease of comparison we represent the zeta potential by its absolute value. The absolute zeta potential is plotted versus surfactant concentration in figure $5 \mathrm{C}$. All surfactants, even at very low concentrations $(<1 \mathrm{~g} \mathrm{~L}$

${ }^{1}$ ), gave absolute zeta potentials of $30 \mathrm{mV}$ or more as required for a stable nanosheet dispersion. As before, all data points cluster together falling close to the same trend line. The zeta potential appears to be roughly constant at $\sim 40 \mathrm{mV}$ for surfactant concentrations between $0.5-10 \mathrm{mM}$. However, it begins to increase above $\mathrm{C}_{\text {surf }} \sim 10 \mathrm{mM}$, reaching $\sim 70 \mathrm{mV}$ for the highest surfactant concentrations. We note that there is a difference in the trend between facial amphiphiles (e.g. SC) and linear amphiphiles (alkyl chain type surfactants e.g. SDS) with a sharper increase in zeta potential with increasing surfactant concentration for the latter. This suggests the adsorption of the two different types of amphiphiles to the nanosheet surface is quite different indeed. 
Other than an increase/decrease in concentration of nanosheets produced, changing the initial concentration of $\mathrm{WS}_{2}$ starting material does not change nanosheet dimensions, Raman or zeta potential results (for details see SI.5). Furthermore changing the head group in the alkyl chain type anionic surfactants does not change concentration, $\langle\mathrm{N}\rangle$ or $\langle\mathrm{L}\rangle$, as illustrated by a comparison of lithium dodecyl sulfate (LDS) and sodium dodecyl sulfate (SDS).

Finally, we note that we observe a trend of increasing zeta potential with decreasing nanosheet dimensions (figure 5D). This may indicate that the adsorbed surfactant density is higher at nanosheet edges compared to the basal plane. This would result in a greater number of surfactant molecules per unit area for smaller nanosheets and so a higher zeta potential. An alternative explanation is that the edges, a source of defects in LPE $\mathrm{WS}_{2}$, are charged in the aqueous environment. Interestingly, in contrast to Gupta et al. ${ }^{25}$ and Varrla et al., ${ }^{62}$ we do not observe a drop in the zeta potential for very high surfactant concentrations due to charge screening effects from the larger pool of counterions.

\section{Destabilization of nanosheets in low and high surfactant concentration}

In order to decouple the effect of stabilization/destabilization from the exfoliation process a sample was exfoliated using a standard surfactant concentration $\left(2 \mathrm{~g} \mathrm{~L}^{-1} \mathrm{SC}\right)$, trapped between $0.1 \mathrm{k} g$ and $21 \mathrm{k} g$ via centrifugation, with the sediment of $21 \mathrm{k} g$ redispersed in low $\left(0.1 \mathrm{~g} \mathrm{~L}^{-1}\right.$ $\mathrm{SC})$ and high (40 $\left.\mathrm{g} \mathrm{L}^{-1} \mathrm{SC}\right)$ surfactant concentrations as shown in Fig 6A. For further details on sample preparation see Methods. The mass of nanosheets, $\langle\mathrm{N}\rangle$ and $\langle\mathrm{L}\rangle$ in redispersed samples are lower compared to the original exfoliated sample (Fig 6B-D). Indeed when this data is compared with the rest of the data set in Fig 4 (blue crosses (X)), the low and high surfactant points appear to fall on the standard mastercurves i.e. where expected for exfoliated samples, rather than only (de)stabilized, in their given surfactant concentrations. There may have been a slight change in the effective surfactant concentration after redispersing which alters the $40 \mathrm{~g} \mathrm{~L}^{-1}$, to larger $\mathrm{N}$ and $\mathrm{L}$ than expected. This data point however remains almost within the scatter of the data and is not of appreciable difference. This result indicates that the choice of surfactant does not necessarily effect the exfoliation of nanosheets during sonication, but has a significant impact on their stabilization. This is in line with previous work which showed that while graphene can be exfoliated by brute force by sonication in water, stabilization only occurs in appropriate liquids. ${ }^{63}$ Additionally, similar results were seen by Fernandes et al. ${ }^{42}$ for CNTs, also noting that the dispersion effectiveness is related to surfactant binding and availability in stabilization rather than exfoliation. The number of stabilized nanosheets is widely independent of surfactant concentration however the dimensions of 
nanosheets changes. Therefore we propose nanosheet nanosheet dimension can tuned by changing the surfactant concentration in which nanosheets are dispersed.

The question remains why the destabilization effect at higher surfactant concentration which causes the observed drop in nanosheet concentration, $\langle\mathrm{L}\rangle$ and $\langle\mathrm{N}\rangle$ happens at a concentration of $\mathrm{C}_{\text {surf }} 10 \mathrm{mM}$. While a fully comprehensive model is well beyond the scope of the manuscript, we suggest that this destabilization is traced down to electrostatic screening. This is confirmed by the data of concentration, $\langle\mathrm{L}\rangle$ and $\langle\mathrm{N}\rangle$ plotted as function of ionic conductivity in SI figure S11.2. While the concentration data is a bit scattered, it is clear that all $\langle\mathrm{L}\rangle$ and $\langle\mathrm{N}\rangle$ data falls on a mastercurve including the nonionic surfactants. The drop in $\langle\mathrm{N}\rangle$ and $\langle\mathrm{L}\rangle$ occurs at an ionic conductivity of $\sim 0.5 \mathrm{mS} / \mathrm{cm}^{-1}$ which seems to be the threshold for electrostatic screening.

\section{Conclusion}

In conclusion, we have found that LPE nanosheets become smaller and thinner once the surfactant concentration exceeds $\sim 10 \mathrm{mM}$. The average number of layers falls as low as 2 with lengths as low as $50 \mathrm{~nm}$ for very high surfactant concentration. The concentration of nanosheets follows a similar trend to nanosheet dimension, falling for $\mathrm{C}_{\text {surf }}>10 \mathrm{mM}$. Raman spectroscopy confirms the volume fraction of monolayers to increase with increasing surfactant concentration. The zeta potential increases with decreasing nanosheet size, probably due to edge effects. We find that only very small concentrations of surfactant $\left(\sim 0.1 \mathrm{~g} \mathrm{~L}^{-1}\right)$ are required for a stable dispersion. In general, all surfactants studied behave in a similar manner with respect to nanosheet exfoliation. As a result, surfactant choice is less important than might be expected. Thus, factors such as cost and environmental effects could become dominant when choosing a suitable surfactant for liquid phase exfoliation.

\section{Methods}

\section{Sample Preparation}

For the preparation of the samples, a two-step sonication procedure was carried out. The first sonication step in water served the purpose to remove impurities that are present in the starting material (see SI section 3). $\mathrm{WS}_{2}, \mathrm{MoS}_{2}$ and graphene dispersions were prepared by sonicating (tapered tip, VibraCell CVX, 750W) powder $\left(\mathrm{WS}_{2}\right.$ Sigma Aldrich $\sim 2 \mu \mathrm{m}, 99 \%, \mathrm{MoS}_{2}$ Sigma Aldrich $\sim 2 \mu \mathrm{m}, 99 \%$, Asbury Graphite grade 3763) at a concentration of $20 \mathrm{~g} \mathrm{~L}^{-1}$ as standard, in $20 \mathrm{~mL}$ of deionised water for $0.5 \mathrm{~h}$ at $25 \%$ amplitude. The dispersion was then centrifuged 
in a Hettich Mikro 220R centrifuge equipped with a fixed-angle rotor 1016 at 3,660 $\mathrm{g}$ for $2 \mathrm{~h}$. The supernatant was discarded and the sediment was redispersed in an aqueous solution of a chosen surfactant (see SI) and concentration. This was subsequently sonicated for $2 \mathrm{~h}$ at $25 \%$ amplitude with a pulse of 6 seconds on and 2 off. The resultant dispersion was centrifuged at $27 \mathrm{~g}$ for $0.5 \mathrm{~h}$ to remove large, unexfoliated material. The sediment was discarded and the supernatant subjected to a trapping between $106 \mathrm{~g}$ and 21,130 $\mathrm{g}$. For centrifugation at low speed (106 $\mathrm{g}$ ), a Hettich Mikro 220R centrifuge equipped with a fixed-angle rotor 1016 was used. The sediment was removed and the supernatant was centrifuged at 21,130 $\mathrm{g}$ in a fixedangle 1195-A rotor. All centrifugation was performed for $2 \mathrm{~h}$ at $10^{\circ} \mathrm{C}$. The supernatant was decanted and the sediment was redispersed in $3 \mathrm{~mL}$ of chosen surfactant and concentration. The final volume of each sample varied slightly due to the variation in the amount of sediment produced. The exact volume was recorded for the calculation of nanosheet mass (see SI section 2.3). This second centrifugation step served the purpose to pellet out the nanosheet to decrease the volume and increase the concentration to facilitate the PL/Raman analysis. In addition to this procedure, two dispersions were prepared with SC $0.1 \mathrm{gL}^{-1}$ and $40 \mathrm{gL}^{-1}$ with a UV Visbased analysis prior to pelleting out the nanosheets in the high speed centrifugation process. The data (see figure 3) is in line with the data obtained using the standard procedure.

For the stabilization test, a standard sample was prepared by sonicating $\mathrm{WS}_{2}$ powder at a concentration of $20 \mathrm{~g} \mathrm{~L}^{-1}$ as standard, in $20 \mathrm{~mL}$ of deionised water for $0.5 \mathrm{~h}$ at $25 \%$ amplitude. The dispersion was then centrifuged at 3,660 $g$ for $2 \mathrm{~h}$. The supernatant was discarded and the sediment was redispersed in an aqueous solution of $2 \mathrm{~g} \mathrm{~L}^{-1} \mathrm{SC}$. This was subsequently sonicated for $2 \mathrm{~h}$ at $25 \%$ amplitude with a pulse of 6 seconds on and 2 off. The resultant dispersion was centrifuged at $27 \mathrm{~g}$ for $0.5 \mathrm{~h}$ to remove large, unexfoliated material. The sediment was discarded and the supernatant subjected to a trapping between $106 \mathrm{~g}$ and 21,130 $\mathrm{g}$. After centrifuging at $21,130 \mathrm{~g}$ the sample was divided in two with half the sediment redispersed in approximately $2 \mathrm{ml}$ of low $\left(0.1 \mathrm{~g} \mathrm{~L}^{-1} \mathrm{SC}\right)$ and high $\left(40 \mathrm{~g} \mathrm{~L}^{-1} \mathrm{SC}\right)$ surfactant concentration respectively. The low and high concentration samples were then centrifuged at $106 g$ for $2 \mathrm{~h}$ to remove aggregated material and the supernatant of each was analyzed with UV-Vis spectroscopy as described below.

\section{Characterisation}

Optical extinction measurements were carried out on a Cary 50 spectrometer in quartz cuvettes in $0.3 \mathrm{~nm}$ increments. A Veeco Nanoscope III-a (Digital Instruments) Atomic Force microscope in tapping mode with HA_HR NT-MDT cantilevers was used for AFM 
measurements. A selection of liquid dispersions $(15 \mu \mathrm{L})$ were diluted until the samples were transparent and drop cast onto preheated $\left(180^{\circ} \mathrm{C}\right) \mathrm{Si} / \mathrm{SiO}_{2}(300 \mathrm{~nm}$ oxide layer) wafers. Brightfield TEM imaging was performed using a JEOL $2100 \mathrm{LaB}$ microscope, operated at $200 \mathrm{kV}$. The dispersion was diluted to optical transparency and manually drop-cast onto a TEM grid with the aim of dropping approximately $0.1 \mathrm{mg}$ of material. One drop at a time was slowly released onto the grid from as close to grid surface as possible to avoid displacing material. The grids were left to dry in air and then placed overnight in a vacuum oven at $70^{\circ} \mathrm{C}$ to dry completely before measuring.

Raman spectroscopy was carried out on an Horiba Jobin Yvon LabRAM HR800 with a 532 $\mathrm{nm}$ excitation laser in air under ambient conditions. The Raman emission was collected by a $100 \times$, long working distance objective lens with $10 \%$ of the laser power. Liquid dispersions were dropped $(\sim 100 \mu \mathrm{L})$ onto glass microscope slides and spectra were measured while the material remained in liquid form. A minimum of 8 spectra at different positions on the liquid drop were recorded and averaged.

Zeta potential and ionic conductivity measurements were carried out on a Malvern Zetasizer Nano system using a $633 \mathrm{~nm} \mathrm{He}-\mathrm{Ne}$ laser. The electrophoretic mobility $(\mu)$ was measured using laser Doppler velocimetry. The electrophoretic mobility is related to the drift velocity of a colloid ( $v$ ) via the applied electric field (E); $v=\mu E$. The zeta potential $\zeta$ is related to the measured electrophoretic mobility $\mu$ using the Smoluchowski approximation for plate-like particles. Folded capillary cells were used for all samples and the measurements were conducted at $20^{\circ} \mathrm{C}$. 10 measurements were taken for each sample with the mean being recorded. The error bar is the standard error of the mean of these measurements. 


\section{Figures}
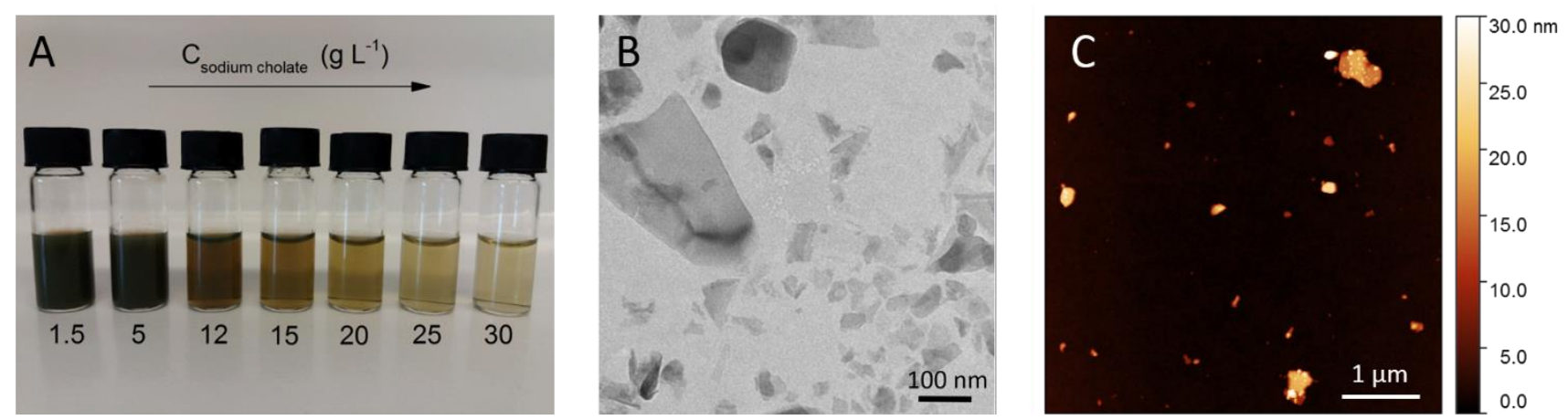

Figure 1: (A) Images of LPE dispersions of $\mathrm{WS}_{2}$ nanosheets with sodium cholate surfactant showing a decrease in nanosheet content with increasing surfactant concentration. Each sample was diluted by a factor of 10 to better show the colour change. (B) Representative TEM image of $\mathrm{WS}_{2}$ nanosheets prepared by exfoliation in sodium cholate surfactant solution $\left(2 \mathrm{~g} \mathrm{~L}^{-1}\right)$. (C) Representative AFM image of $\mathrm{WS}_{2}$ nanosheets exfoliated in sodium cholate $\left(2 \mathrm{~g} \mathrm{~L}^{-1}\right)$. Each image shows a broad range of nanosheet sizes and thicknesses. 

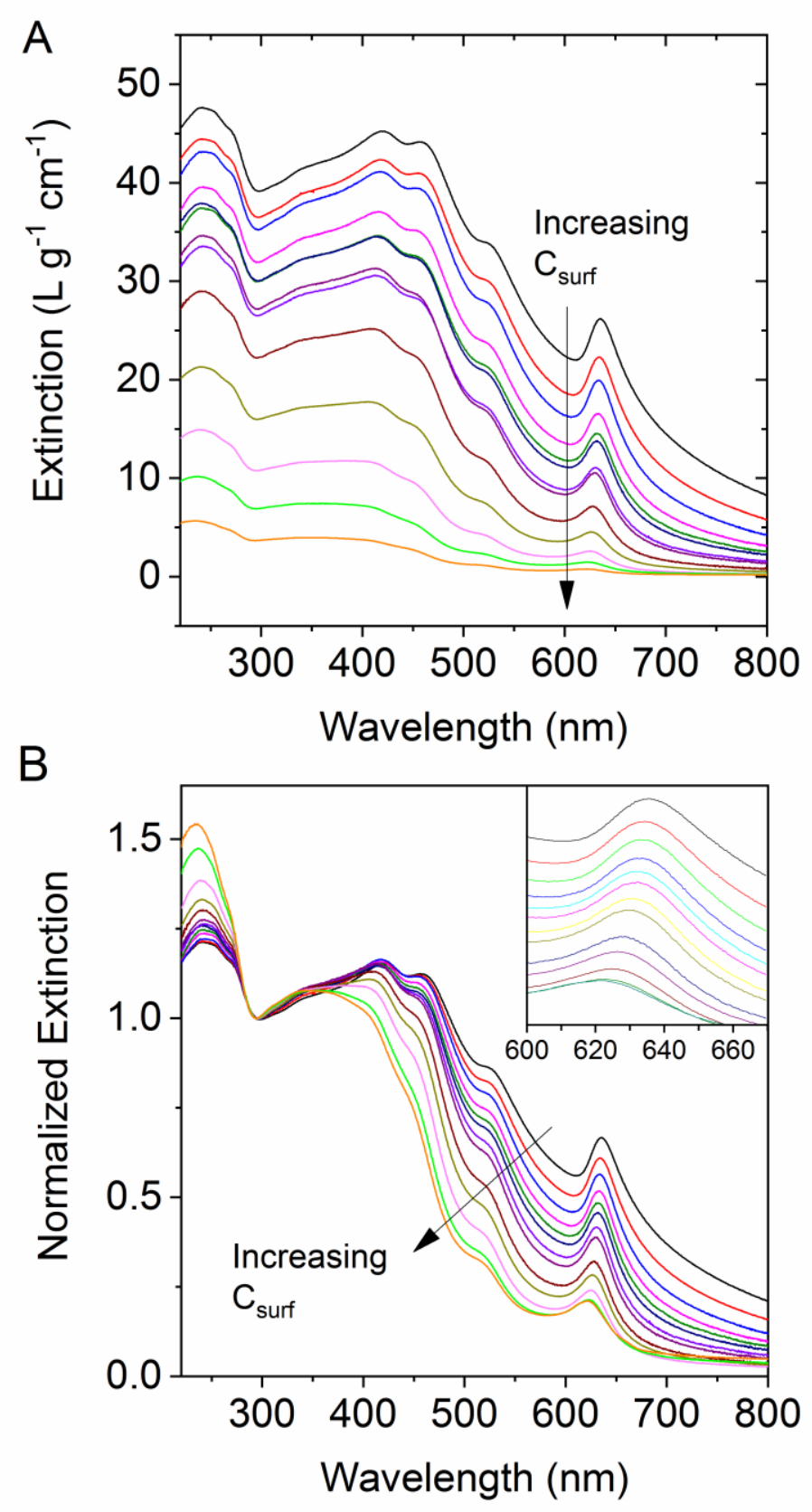

Figure 2: (A) Optical extinction spectra of dispersions of $\mathrm{WS}_{2}$ nanosheets exfoliated in aqueous sodium cholate surfactant solutions with a range of surfactant concentrations from 0.2 to $25 \mathrm{~g} \mathrm{~L}^{-1}(0.46$ to $58 \mathrm{mM})$. (B) The same spectra as in A, normalized to the extinction value at $294 \mathrm{~nm}$. Inset: magnified view of A-exciton region. 


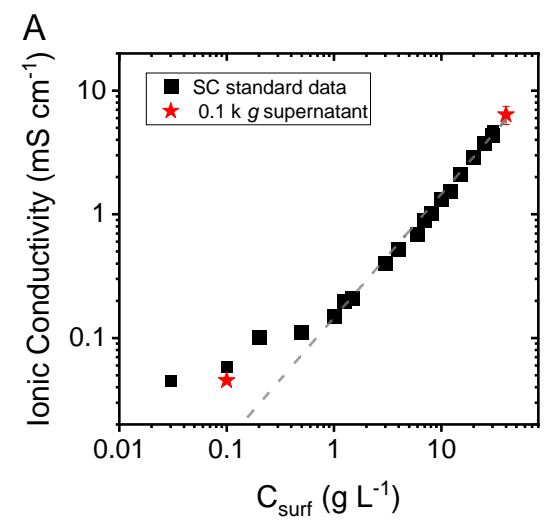

B
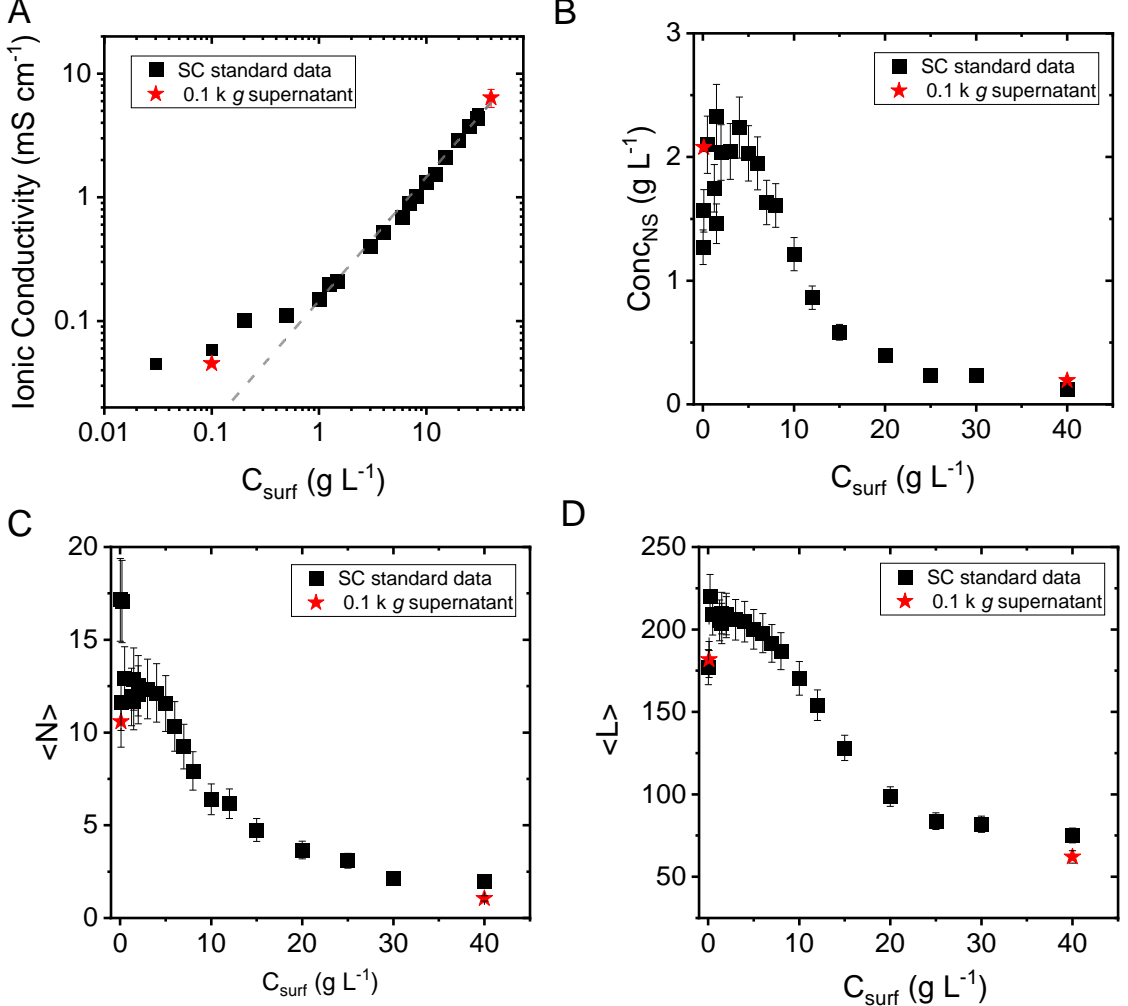

Figure 3: (A) Ionic conductivities of $\mathrm{WS}_{2}-\mathrm{SC}$ dispersions as function of SC concentration. The dashed line it a linear fit to the though the origin illustrating that the effect of ionic impurities will only affect data at low surfactant concentration. (B) Concentration of $\mathrm{WS}_{2}$ nanosheets as a function of SC concentration expressed in $\mathrm{g} \mathrm{L}^{-1}$ no a linear scale. (C-D) Mean layer number $\langle\mathrm{N}\rangle$ (C) and mean nanosheet length $\langle\mathrm{L}\rangle$ (D) versus SC concentration. The red stars are samples where the centrifugation step at high speeds (required for further PL/Raman measurements) was omitted. 

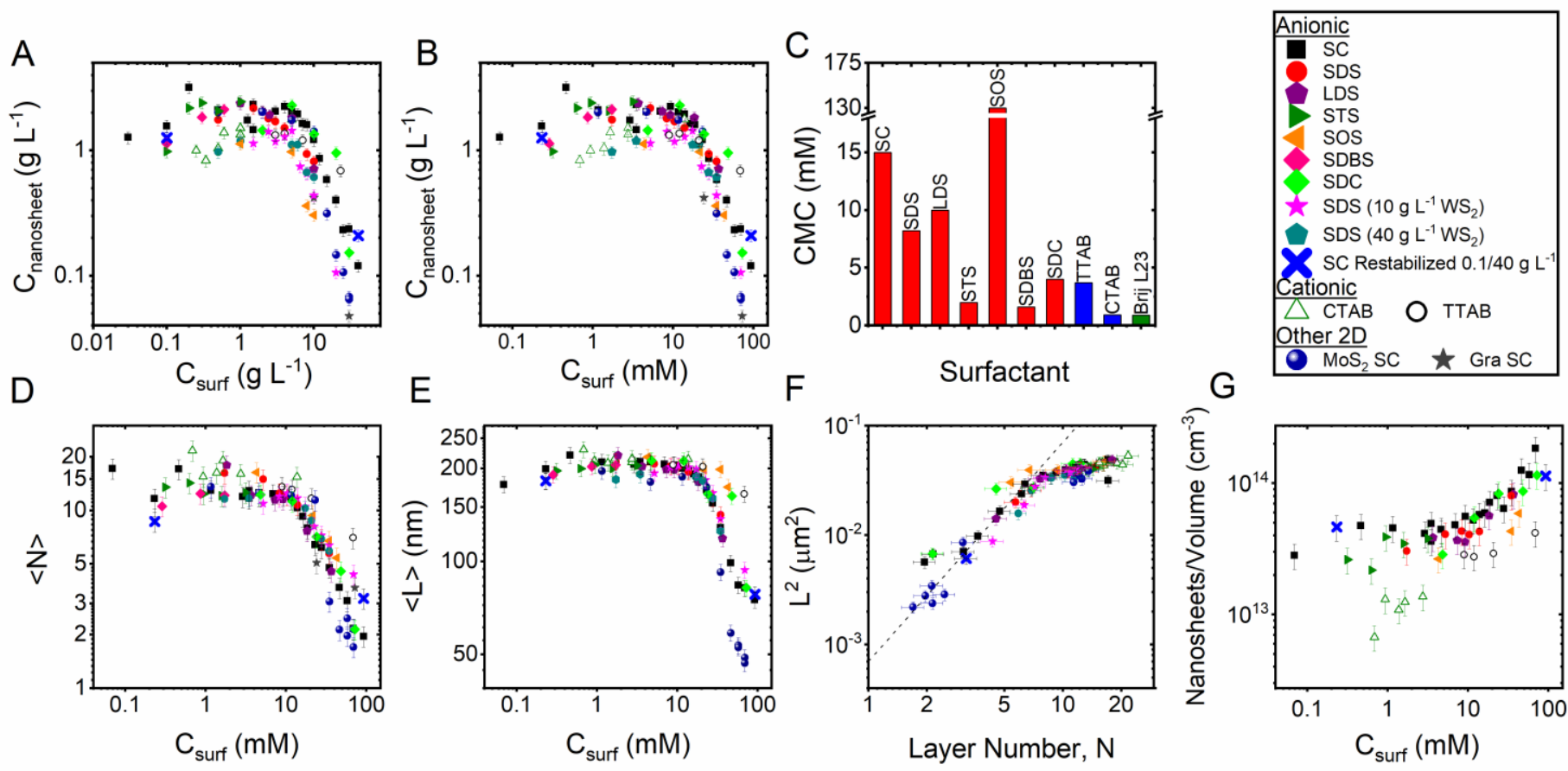

Figure 4: (A) Concentration of $\mathrm{WS}_{2}$ nanosheets as a function of surfactant concentration expressed in $\mathrm{g} \mathrm{L}^{-1}$ for a range of ionic surfactants. (B) The same data as A with surfactant concentration expressed in mM. (C) Bar chart of the critical micelle concentration (CMC) $(\mathrm{mM})$ of each surfactant with anionics (red), cationics (blue) and non-ionic (green). (D-E) Mean layer number $\langle\mathrm{N}\rangle$ and mean nanosheet length $\langle\mathrm{L}\rangle$ versus surfactant concentration. $(\mathrm{F})$ Square of mean nanosheet length plotted versus layer number. As expected, $\langle\mathrm{L}\rangle^{2}$ vs $\langle\mathrm{N}\rangle$ follows a power law up to $\langle\mathrm{N}\rangle \sim 10$ (dashed line). The deviation above this indicates the metrics are no longer fully reliable in the range. $(\mathrm{G})$ Total number of nanosheets per volume of initial exfoliated dispersion ( $20 \mathrm{ml}$ ) versus surfactant concentration. Top Right: Symbol legend for each surfactant for the data throughout. 

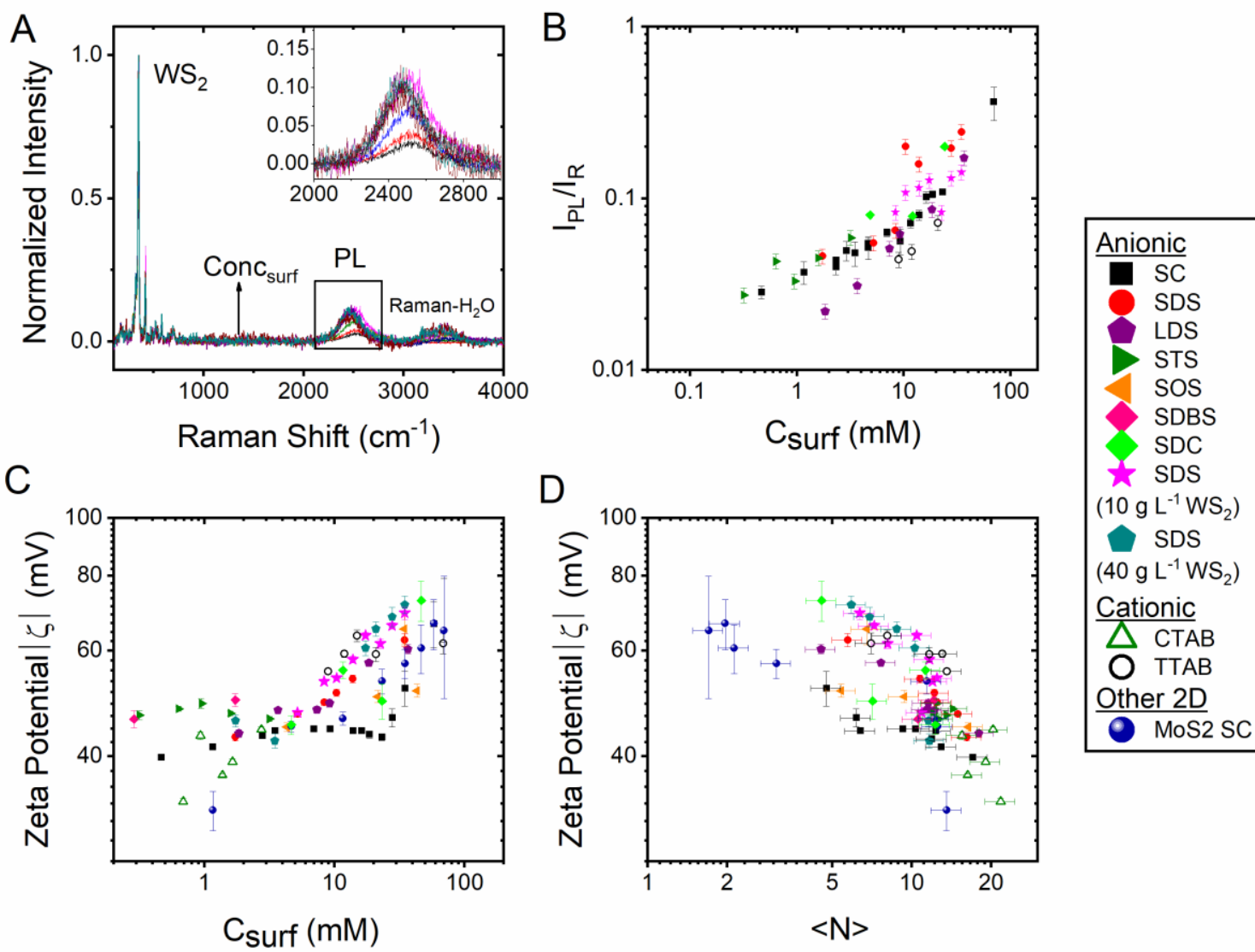

Figure 5: (A) Examples of Raman spectra $\left(\lambda_{\mathrm{ex}}=532 \mathrm{~nm}\right)$ for $\mathrm{WS}_{2}$ nanosheets in sodium cholate surfactant at a range of concentrations normalized to the $\mathrm{WS}_{2}$ Raman mode at $\sim 350 \mathrm{~cm}^{-1}$. The peak at $\sim 2500 \mathrm{~cm}^{-1}$ is photoluminescence from $\mathrm{WS}_{2}$ which increases with increasing surfactant concentration (inset). (B) Ratio of the intensities of PL peak and $\mathrm{WS}_{2}$ Raman peak $\left(\sim 350 \mathrm{~cm}^{-}\right.$ $\left.{ }^{1}\right)$ versus surfactant concentration. (C) A plot of the absolute value of zeta potential measurements versus surfactant concentration. (D) A plot of the absolute value of zeta potential measurements versus mean nanosheet length $\langle\mathrm{L}\rangle$. 
A

Sonicated $2 \mathrm{~g} \mathrm{~L}^{-1} \mathrm{SC}$

$0.1 \mathrm{~kg}$ supernatant
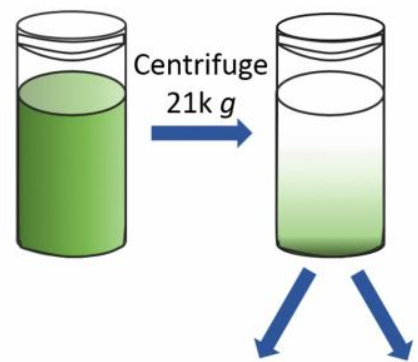

Redisperse $0.1 \mathrm{~g} \mathrm{~L}^{-1} \mathrm{SC}$

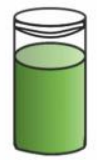

Centrifuge

$0.1 \mathrm{~kg}$,

Analyze

C

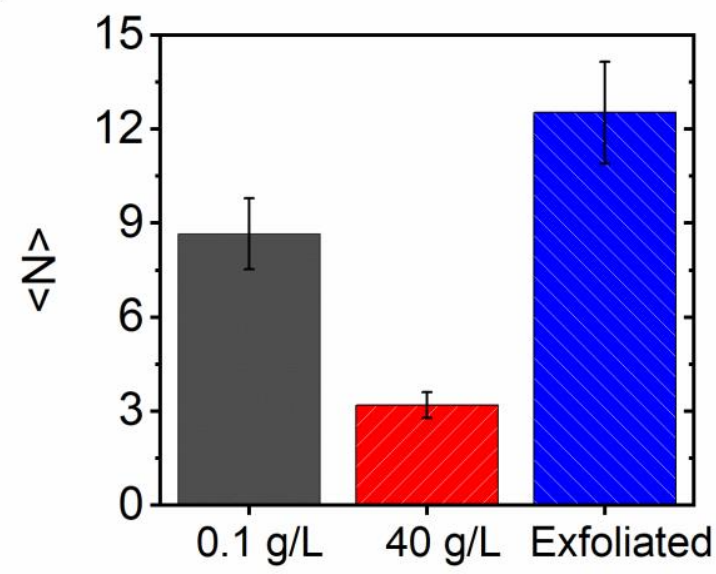

B
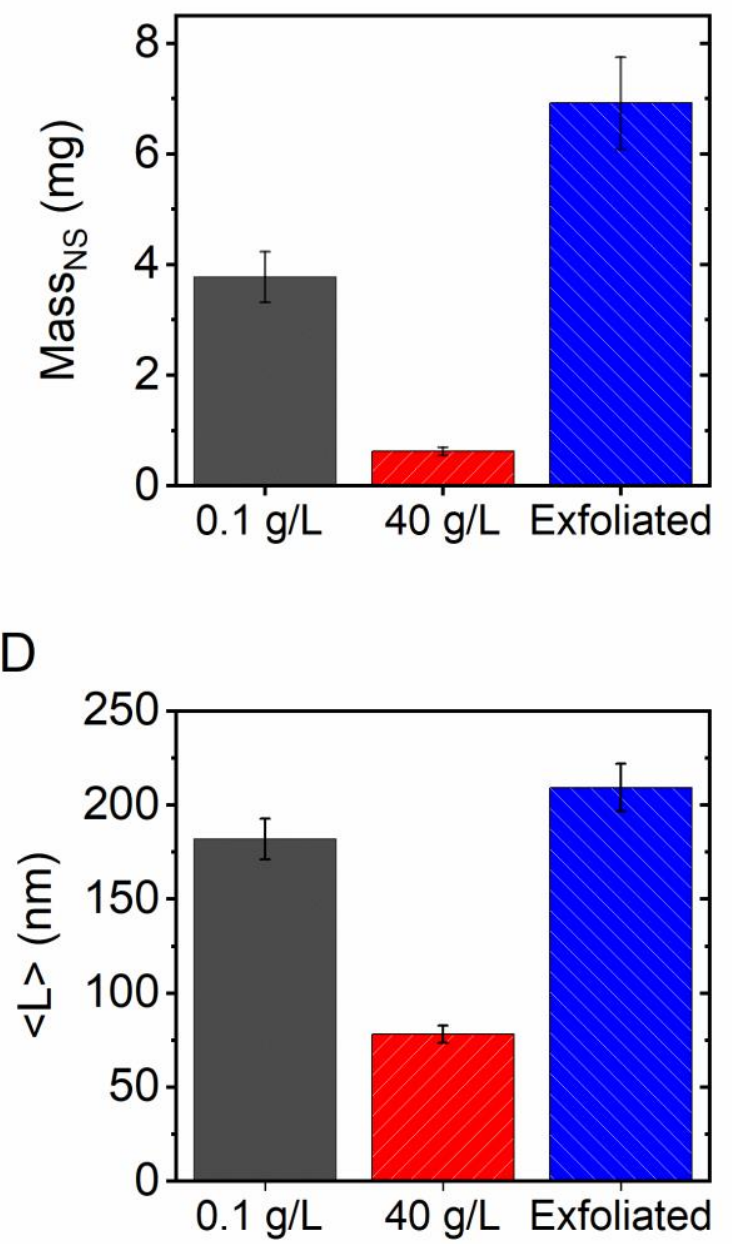

Figure 6: (A) Schematic of the experiment to test the stabilization of nanosheets. An exfoliated $\mathrm{WS}_{2}$ sample in $2 \mathrm{~g} \mathrm{~L}^{-1} \mathrm{SC}$ was prepared, trapped $0.1-21 \mathrm{k} g$ with the $21 \mathrm{k} g$ sediment redispersed in low $\left(0.1 \mathrm{~g} \mathrm{~L}^{-1}\right)$ and high $\left(40 \mathrm{~g} \mathrm{~L}^{-1}\right)$ surfactant concentration. The samples were re-centrifuged at $0.1 \mathrm{k} g$ to remove aggregated material. (B) Bar chart of nanosheet mass for each surfactant concentration. The calculated mass was multiplied by a factor of 2 to take into account that the original dispersion was divided in half for redispersing. (C) Bar chart of mean layer number $\langle\mathrm{N}\rangle$ for each surfactant concentration (D) Bar chart of mean nanosheet $\langle\mathrm{L}\rangle$ for each surfactant concentration. 


\section{Acknowledgement}

We acknowledge the European Union under grant agreements $n^{\circ} 785219$ Graphene Flagshipcore 2 and the European Research Council Advanced Grant (FUTURE-PRINT). C.B. acknowledges the German research foundation DFG under Emmy-Noether grant BA4856/2-2

\section{Additional information}

Supplementary information is available and includes; surfactant list, details on metrics, additional sample preparation information, reproducibility and error analysis, comparison by nonionic surfactant data, surfactant type, ionic conductivity data.

\section{For Table of Contents Only}

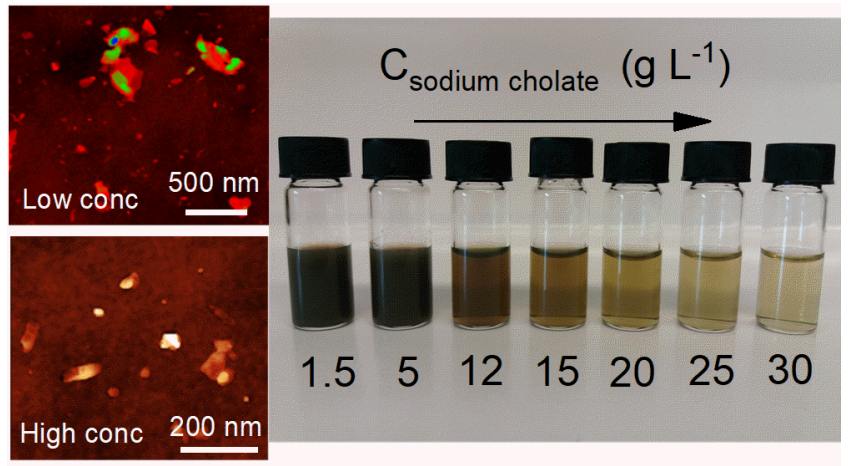

\section{References}

1. Bonaccorso, F.; Bartolotta, A.; Coleman, J. N.; Backes, C., 2D-Crystal-Based Functional Inks. Adv. Mater. 2016, 28, (29), 6136-6166.

2. Hernandez, Y.; Nicolosi, V.; Lotya, M.; Blighe, F. M.; Sun, Z.; De, S.; McGovern, I.; Holland, B.; Byrne, M.; Gun'Ko, Y. K., High-yield production of graphene by liquid-phase exfoliation of graphite. Nat. Nanotechnol. 2008, 3, (9), 563.

3. $\quad$ Backes, C.; Paton, K. R.; Hanlon, D.; Yuan, S.; Katsnelson, M. I.; Houston, J.; Smith, R. J.; McCloskey, D.; Donegan, J. F.; Coleman, J. N., Spectroscopic metrics allow in situ measurement of mean size and thickness of liquid-exfoliated few-layer graphene nanosheets. Nanoscale 2016, 8, (7), 4311-4323.

4. Hanlon, D.; Backes, C.; Doherty, E.; Cucinotta, C. S.; Berner, N. C.; Boland, C.; Lee, K.; Harvey, A.; Lynch, P.; Gholamvand, Z., Liquid exfoliation of solvent-stabilized few-layer black phosphorus for applications beyond electronics. Nat Commun 2015, 6, 8563.

5. $\quad$ Backes, C.; Smith, R. J.; McEvoy, N.; Berner, N. C.; McCloskey, D.; Nerl, H. C.; O’Neill, A.; King, P. J.; Higgins, T.; Hanlon, D., Edge and confinement effects allow in situ measurement of size and thickness of liquid-exfoliated nanosheets. Nat Commun 2014, 5, 4576. 
6. Boland, J. B.; Harvey, A.; Tian, R.; Hanlon, D.; Vega-Mayoral, V.; Szydlowska, B.; Griffin, A.; Stimpel-Lindner, T.; Jaskaniec, S.; Nicolosi, V., Liquid phase exfoliation of MoO 2 nanosheets for lithium ion battery applications. Nanoscale Adv 2019, 1, 1560-1570.

7. $\quad$ Griffin, A.; Harvey, A.; Cunningham, B.; Scullion, D.; Tian, T.; Shih, C.-J.; Gruening, M.; Donegan, J. F.; Santos, E. J.; Backes, C., Spectroscopic size and thickness metrics for liquidexfoliated h-BN. Chem. Mater. 2018, 30, (6), 1998-2005.

8. Hernandez, Y.; Nicolosi, V.; Lotya, M.; Blighe, F. M.; Sun, Z. Y.; De, S.; McGovern, I. T.; Holland, B.; Byrne, M.; Gun'ko, Y. K.; Boland, J. J.; Niraj, P.; Duesberg, G.; Krishnamurthy, S.; Goodhue, R.; Hutchison, J.; Scardaci, V.; Ferrari, A. C.; Coleman, J. N., High-yield production of graphene by liquid-phase exfoliation of graphite. Nat. Nanotechnol. 2008, 3, (9), 563-568.

9. Liu, L. H.; Zorn, G.; Castner, D. G.; Solanki, R.; Lerner, M. M.; Yan, M. D., A simple and scalable route to wafer-size patterned graphene. J. Mater. Chem. 2010, 20, (24), 5041-5046.

10. Du, W. C.; Jiang, X. Q.; Zhu, L. H., From graphite to graphene: direct liquid-phase exfoliation of graphite to produce single- and few-layered pristine graphene. J Mater Chem A 2013, 1, (36), 10592-10606.

11. Zhi, C. Y.; Bando, Y.; Tang, C. C.; Kuwahara, H.; Golberg, D., Large-Scale Fabrication of Boron Nitride Nanosheets and Their Utilization in Polymeric Composites with Improved Thermal and Mechanical Properties. Adv. Mater. 2009, 21, (28), 2889-+.

12. Harvey, A.; Backes, C.; Gholamvand, Z.; Hanlon, D.; McAteer, D.; Nerl, H. C.; McGuire, E.; Seral-Ascaso, A.; Ramasse, Q. M.; McEvoy, N.; Winters, S.; Berner, N. C.; McCloskey, D.; Donegan, J. F.; Duesberg, G. S.; Nicolosi, V.; Coleman, J. N., Preparation of Gallium Sulfide Nanosheets by Liquid Exfoliation and Their Application As Hydrogen Evolution Catalysts. Chem. Mater. 2015, 27 , (9), 3483-3493.

13. Hanlon, D.; Backes, C.; Doherty, E.; Cucinotta, C. S.; Berner, N. C.; Boland, C.; Lee, K.; Harvey, A.; Lynch, P.; Gholamvand, Z.; Zhang, S. F.; Wang, K. P.; Moynihan, G.; Pokle, A.; Ramasse, Q. M.; McEvoy, N.; Blau, W. J.; Wang, J.; Abellan, G.; Hauke, F.; Hirsch, A.; Sanvito, S.; O'Regan, D. D.; Duesberg, G. S.; Nicolosi, V.; Coleman, J. N., Liquid exfoliation of solventstabilized few-layer black phosphorus for applications beyond electronics. Nat Commun 2015, 6. 14. Naguib, M.; Mashtalir, O.; Carle, J.; Kurtoglu, M.; Presser, V.; Lu, J.; Hultman, L.; Gogotsi, Y.; Barsoum, M. W., MXenes: A new family of 2D early transition metal carbides produced by exfoliation of the MAX phases. Abstr Pap Am Chem S 2012, 244.

15. Coleman, J. N.; Lotya, M.; O'Neill, A.; Bergin, S. D.; King, P. J.; Khan, U.; Young, K.; Gaucher, A.; De, S.; Smith, R. J.; Shvets, I. V.; Arora, S. K.; Stanton, G.; Kim, H. Y.; Lee, K.; Kim, G. T.; Duesberg, G. S.; Hallam, T.; Boland, J. J.; Wang, J. J.; Donegan, J. F.; Grunlan, J. C.; Moriarty, G.; Shmeliov, A.; Nicholls, R. J.; Perkins, J. M.; Grieveson, E. M.; Theuwissen, K.; McComb, D. W.; Nellist, P. D.; Nicolosi, V., Two-Dimensional Nanosheets Produced by Liquid Exfoliation of Layered Materials. Science 2011, 331, (6017), 568-571.

16. Bang, G. S.; Nam, K. W.; Kim, J. Y.; Shin, J.; Choi, J. W.; Choi, S. Y., Effective LiquidPhase Exfoliation and Sodium Ion Battery Application of MoS2 Nanosheets. Acs Applied Materials \& Interfaces 2014, 6, (10), 7084-7089.

17. Lotya, M.; King, P. J.; Khan, U.; De, S.; Coleman, J. N., High-concentration, surfactantstabilized graphene dispersions. ACS nano 2010, 4, (6), 3155-3162.

18. Tummala, N. R.; Striolo, A., Role of Counterion Condensation in the Self-Assembly of SDS Surfactants at the Water- Graphite Interface. J. Phys. Chem. B 2008, 112, (7), 1987-2000.

19. Buzaglo, M.; Shtein, M.; Kober, S.; Lovrinčić, R.; Vilan, A.; Regev, O., Critical parameters in exfoliating graphite into graphene. PCCP 2013, 15, (12), 4428-4435.

20. Lee, Y. J.; Huang, L.; Wang, H.; Sushko, M. L.; Schwenzer, B.; Aksay, I. A.; Liu, J., Structural rearrangement and dispersion of functionalized graphene sheets in aqueous solutions. Colloids Interfac. Sci. 2015, 8, 1-5.

21. Poorsargol, M.; Sohrabi, B.; Dehestani, M., Study of the Gemini Surfactants' Self-Assembly on Graphene Nanosheets: Insights from Molecular Dynamic Simulation. J. Phys. Chem. A 2018, 122, (15), 3873-3885.

22. Wang, S.; Yi, M.; Shen, Z., The effect of surfactants and their concentration on the liquid exfoliation of graphene. RSC Adv. 2016, 6, (61), 56705-56710. 
23. Gupta, A.; Arunachalam, V.; Vasudevan, S., Water dispersible, positively and negatively charged MoS2 nanosheets: surface chemistry and the role of surfactant binding. J. Phys. Chem. Lett 2015, 6, (4), 739-744.

24. Lin, S.; Shih, C.-J.; Strano, M. S.; Blankschtein, D., Molecular insights into the surface morphology, layering structure, and aggregation kinetics of surfactant-stabilized graphene dispersions. J. Am. Chem. Soc. 2011, 133, (32), 12810-12823.

25. Gupta, A.; Vasudevan, S., Understanding Surfactant Stabilization of MoS2 Nanosheets in Aqueous Dispersions from Zeta Potential Measurements and Molecular Dynamics Simulations. $J$. Phys. Chem. C 2018, 122, (33), 19243-19250.

26. Ramalingam, P.; Pusuluri, S. T.; Periasamy, S.; Veerabahu, R.; Kulandaivel, J., Role of deoxy group on the high concentration of graphene in surfactant/water media. $R S C A d v . \mathbf{2 0 1 3}, 3,(7), 2369$ 2378.

27. Vera-López, S.; Martínez, P.; San Andrés, M.; Díez-Pascual, A.; Valiente, M., Study of graphene dispersions in sodium dodecylsulfate by steady-state fluorescence of pyrene. J. Colloid Interface Sci. 2018, 514, 415-424.

28. Israelachvili, J. N., Intermolecular and surface forces. San Diego: Academic press, 2015.

29. Bhattacharya, S.; Samanta, S. K., Surfactants possessing multiple polar heads. A perspective on their unique aggregation behavior and applications. J. Phys. Chem. Lett 2011, 2, (8), 914-920.

30. Bogdanova, L.; Gnezdilov, O.; Idiyatullin, B.; Kurbanov, R. K.; Zuev, Y. F.; Us’yarov, O., Micellization in sodium deoxycholate solutions. Colloid J. 2012, 74, (1), 1-6.

31. Pártay, L. B.; Jedlovszky, P.; Sega, M., Molecular aggregates in aqueous solutions of bile acid salts. Molecular dynamics simulation study. J. Phys. Chem. B 2007, 111, (33), 9886-9896.

32. Backes, C.; Szydłowska, B. M.; Harvey, A.; Yuan, S.; Vega-Mayoral, V.; Davies, B. R.; Zhao, P.-1.; Hanlon, D.; Santos, E. J. G.; Katsnelson, M. I.; Blau, W. J.; Gadermaier, C.; Coleman, J. N., Production of Highly Monolayer Enriched Dispersions of Liquid-Exfoliated Nanosheets by Liquid Cascade Centrifugation. ACS Nano 2016, 10 (1), 1589-1601.

33. White, B.; Banerjee, S.; O'Brien, S.; Turro, N. J.; Herman, I. P., Zeta-potential measurements of surfactant-wrapped individual single-walled carbon nanotubes. J. Phys. Chem. C 2007, 111, (37), 13684-13690.

34. Clark, M. D.; Subramanian, S.; Krishnamoorti, R., Understanding surfactant aided aqueous dispersion of multi-walled carbon nanotubes. J. Colloid Interface Sci. 2011, 354, (1), 144-151. 35. Blanch, A. J.; Lenehan, C. E.; Quinton, J. S., Optimizing surfactant concentrations for dispersion of single-walled carbon nanotubes in aqueous solution. J. Phys. Chem. B 2010, 114, (30), 9805-9811.

36. Xu, Z.; Yang, X.; Yang, Z., A molecular simulation probing of structure and interaction for supramolecular sodium dodecyl sulfate/single-wall carbon nanotube assemblies. Nano Lett. 2010, 10, (3), 985-991.

37. Islam, M.; Rojas, E.; Bergey, D.; Johnson, A.; Yodh, A., High weight fraction surfactant solubilization of single-wall carbon nanotubes in water. Nano Lett. 2003, 3, (2), 269-273.

38. Angelikopoulos, P.; Bock, H., The science of dispersing carbon nanotubes with surfactants. PCCP 2012, 14, (27), 9546-9557.

39. Strano, M. S.; Moore, V. C.; Miller, M. K.; Allen, M. J.; Haroz, E. H.; Kittrell, C.; Hauge, R. H.; Smalley, R., The role of surfactant adsorption during ultrasonication in the dispersion of singlewalled carbon nanotubes. J. Nanosci. Nanotechnol 2003, 3, (1-2), 81-86.

40. Wang, H., Dispersing carbon nanotubes using surfactants. Current Opinion in Colloid \& Interface Science 2009, 14, (5), 364-371.

41. Utsumi, S.; Kanamaru, M.; Honda, H.; Kanoh, H.; Tanaka, H.; Ohkubo, T.; Sakai, H.; Abe, M.; Kaneko, K., RBM band shift-evidenced dispersion mechanism of single-wall carbon nanotube bundles with NaDDBS. J. Colloid Interface Sci. 2007, 308, (1), 276-284.

42. Fernandes, R. M.; Abreu, B.; Claro, B.; Buzaglo, M.; Regev, O.; Furó, I. n.; Marques, E. F., Dispersing carbon nanotubes with ionic surfactants under controlled conditions: comparisons and insight. Langmuir 2015, 31, (40), 10955-10965.

43. Vigolo, B.; Penicaud, A.; Coulon, C.; Sauder, C.; Pailler, R.; Journet, C.; Bernier, P.; Poulin, P., Macroscopic fibers and ribbons of oriented carbon nanotubes. Science 2000, 290, (5495), 13311334. 
44. Bonard, J. M.; Stora, T.; Salvetat, J. P.; Maier, F.; Stöckli, T.; Duschl, C.; Forró, L.; de Heer, W. A.; Châtelain, A., Purification and size-selection of carbon nanotubes. Adv. Mater. 1997, 9, (10), 827-831.

45. Wang, H.; Zhou, W.; Ho, D. L.; Winey, K. I.; Fischer, J. E.; Glinka, C. J.; Hobbie, E. K., Dispersing single-walled carbon nanotubes with surfactants: a small angle neutron scattering study.

Nano Lett. 2004, 4, (9), 1789-1793.

46. Vaisman, L.; Wagner, H. D.; Marom, G., The role of surfactants in dispersion of carbon nanotubes. Adv. Colloid Interface Sci. 2006, 128, 37-46.

47. Schlierf, A.; Yang, H.; Gebremedhn, E.; Treossi, E.; Ortolani, L.; Chen, L.; Minoia, A.; Morandi, V.; Samorì, P.; Casiraghi, C., Nanoscale insight into the exfoliation mechanism of graphene with organic dyes: effect of charge, dipole and molecular structure. Nanoscale 2013, 5, (10), 42054216.

48. Parviz, D.; Das, S.; Ahmed, H. T.; Irin, F.; Bhattacharia, S.; Green, M. J., Dispersions of noncovalently functionalized graphene with minimal stabilizer. Acs Nano 2012, 6, (10), 8857-8867.

49. Just-Baringo, X.; Shin, Y.; Zarattini, M.; Nagyte, V.; Zhao, L.; Kostarelos, K.; Casiraghi, C.; Larrosa, I., Palladium Catalysed C-H Arylation of Pyrenes: Access to a New Class of Exfoliating Agents for Water-Based Graphene Dispersions. Chem. Sci. 2020, 11, 2472-2478.

50. Backes, C.; Campi, D.; Szydłowska, B. M.; Synnatschke, K.; Ojala, E.; Rashvand, F.; Harvey, A.; Griffin, A.; Sofer, Z.; Marzari, N.; Coleman, J. N.; O’Regan, D. D., Equipartition of Energy Defines the Size-Thickness Relationship in Liquid-Exfoliated Nanosheets. ACS Nano 2019, 13, (6), 7050-7061.

51. Backes, C.; Szydłowska, B. M.; Harvey, A.; Yuan, S.; Vega-Mayoral, V.; Davies, B. R.; Zhao, P.-1.; Hanlon, D.; Santos, E. J.; Katsnelson, M. I., Production of highly monolayer enriched dispersions of liquid-exfoliated nanosheets by liquid cascade centrifugation. ACS nano 2016, 10, (1), 1589-1601.

52. Smith, R. J.; Lotya, M.; Coleman, J. N., The importance of repulsive potential barriers for the dispersion of graphene using surfactants. New J. Phys. 2010, 12, (12), 125008.

53. Nakato, T.; Kawamata, J.; Takagi, S., Inorganic Nanosheets and Nanosheet-Based Materials. Japan: Springer, 2017.

54. Park, K.; Koerner, H.; Vaia, R. A., Depletion-induced shape and size selection of gold nanoparticles. Nano Lett. 2010, 10, (4), 1433-1439.

55. Evans, H., 117. Alkyl sulphates. Part I. Critical micelle concentrations of the sodium salts. Journal of the Chemical Society (Resumed) 1956, 579-586.

56. Sepulveda, L.; Cortés, J., Ionization degrees and critical micelle concentrations of hexadecyltrimethylammonium and tetradecyltrimethylammonium micelles with different counterions. J. Phys. Chem. 1985, 89, (24), 5322-5324.

57. Djamil, J.; Hansen, A.-L.; Backes, C.; Bensch, W.; Schürmann, U.; Kienle, L.; Düvel, A.; Heitjans, P., Using light, X-rays and electrons for evaluation of the nanostructure of layered materials. Nanoscale 2018, 10, (45), 21142-21150.

58. Poorsargol, M.; Alimohammadian, M.; Sohrabi, B.; Dehestani, M., Dispersion of graphene using surfactant mixtures: Experimental and molecular dynamics simulation studies. Appl. Surf. Sci. 2019, 464, 440-450.

59. Davey, T. W.; Ducker, W. A.; Hayman, A. R.; Simpson, J., Krafft temperature depression in quaternary ammonium bromide surfactants. Langmuir 1998, 14, (12), 3210-3213.

60. Berkdemir, A.; Gutiérrez, H. R.; Botello-Méndez, A. R.; Perea-López, N.; Elías, A. L.; Chia, C.-I.; Wang, B.; Crespi, V. H.; López-Urías, F.; Charlier, J.-C., Identification of individual and few layers of WS 2 using Raman spectroscopy. Sci. Rep 2013, 3, 1755.

61. Ueberricke, L.; Coleman, J. N.; Backes, C., Robustness of Size Selection and Spectroscopic Size, Thickness and Monolayer Metrics of Liquid-Exfoliated WS2. Phys. Status Solidi B 2017, 254, (11), 1700443.

62. Varrla, E.; Backes, C.; Paton, K. R.; Harvey, A.; Gholamvand, Z.; McCauley, J.; Coleman, J. N., Large-scale production of size-controlled MoS2 nanosheets by shear exfoliation. Chem. Mater. 2015, 27, (3), 1129-1139. 
63. Barwich, S.; Khan, U.; Coleman, J. N., A technique to pretreat graphite which allows the rapid dispersion of defect-free graphene in solvents at high concentration. J. Phys. Chem. C 2013, 117, (37), 19212-19218. 